\title{
State-of-Charge Estimation of Lithium-ion Battery Based on a Novel Reduced Order Electrochemical Model
}

\author{
Yuan Chaochun, Wang Bingjian, Zhang Houzhong, Long Chen, Li Huanhuan*
}

Automotive Engineering Research Institute, Jiangsu University, 301 Xuefu Road, Zhenjiang 212013, China

*E-mail: lihh@mail.ujs.edu.cn

doi: $10.20964 / 2018.01 .133$

Received: 15 September 2017 / Accepted: 12 December 2017 / Published: 16 December 2017

On the basis of traditional single-particle model, an extended single-particle (ESP) electrochemical model that considers the influence of electrolyte phase potential on terminal voltage in the light of electrochemical characteristics of lithium ion battery is designed. The linear decreasing weight particle swarm algorithm is adopted to identify the key parameters of the ESP electrochemical model to reduce the effect of parameter identification error on the accuracy of state-of-charge (SOC) estimation. And an ESP-model-based extended Kalman filter (EKF) algorithm which can compensate the error caused by the simplified solution and random noise by feedback control is also proposed. The simulation results demonstrate that EKF algorithm reduce calculation errors, parameter measurement noises and increase accuracy of SOC estimation for lithium ion battery. Finally, the Charge/discharge test using $2300 \mathrm{mAh} \mathrm{LiFePO}_{4}$ battery is carried out at 3C FUDS and comparison of experimental and simulated results show that ESP-model-based EKF algorithm using for SOC estimation has good accuracy and robustness.

Keywords: Lithium-ion Battery; SOC Estimation Algorithm; Extended single-particle model; Model Errors

\section{$\underline{\text { FULL TEXT }}$}

(C) 2018 The Authors. Published by ESG (www.electrochemsci.org). This article is an open access article distributed under the terms and conditions of the Creative Commons Attribution license (http://creativecommons.org/licenses/by/4.0/). 